\title{
Continuity of derivations on topological algebras of power series
}

\author{
R. J. Loy
}

\begin{abstract}
Let $A$ be an algebra of formal power series in one indeterminate over the complex field, $D$ a derivation on $A$. It is shown that if $A$ has a Fréchet space topology under which it is a topological algebra, then $D$ is necessarily continuous provided the coordinate projections satisfy a certain equicontinuity condition. This condition is always satisfied if $A$ is a Banach algebra and the projections are continuous. A second result is given, with weaker hypothesis on the projections and correspondingly weaker conclusion.
\end{abstract}

In this paper we show that derivations on a certain class of metrizable topological algebras of formal power series are necessarily continuous. This result is contained in one of Johnson [3], however the proof as outlined in that paper requires the algebras to satisfy a certain algebraic condition which we do not assume here. Algebras satisfying this condition are considered in [4], where it is shown that they are necessarily semisimple in the Banach algebra case. The class we consider here, however, contains non-semisimple Banach algebras. Indeed, we consider a particular case below of a radical Banach Algebra.

Throughout this paper $A$ will denote an algebra of formal power series in an indeterminate $t$ over the complex field, containing the element $t$, but not necessarily having an identity. Elements of $A$ will be denoted by expressions of the form $\sum a_{i} t^{i}$. We suppose further that $A$ is a

Received 11 August 1969. This research was supported by a Post-Doctoral Fellowship at Carleton University.

419 
topological algebra under a complete locally convex metrizable topology determined by a sequence $\left\{\|\cdot\|_{n}\right\}_{n \geq 1}$ of seminorms, and note that multiplication in $A$ is necessarily jointly continuous ([1], Theorem 5).

THEOREM 1. Suppose that the projections $p_{j}: \sum a_{i} t^{i}+a_{j}$ are continuous and, moreover, that there is a sequence $\left\{\gamma_{n}\right\}_{n \geq 0}$ of positive real numbers such that the family $\left\{\gamma_{n}^{-1} p_{n}\right\}_{n \geq 0}$ is equicontinuous. Then any derivation on $A$ is continuous.

Proof. Suppose to the contrary that $D$ is a discontinuous derivation on $A$. Since $\left\{p_{n}\right\}_{n \geq 0}$ is a separating family of continuous linear functionals on $A$ it follows by the closed graph theorem that at least one of the functionals $\left\{p_{n} D\right\}_{n \geq 0}$, and hence one with least index, $p_{k} D$ say, is discontinuous. In order to give a proof valid for all values of $k$ we make the convention that empty sums, of the form $\sum_{i=1}^{j}(\cdot)_{i}$ with $j<1$, have the value zero.

Since the functionals $p_{0} D, \ldots, p_{k-1} D$ are continuous there is a neighbourhood $U$ of zero in $A$ such that if $x \in U$ then $\left|p_{j}(D x)\right| \leq 1$, $0 \leq j \leq k-1$. Also, by the equicontinuity of the family $\left\{\gamma_{n}^{-1} p_{n}\right\}_{n \geq 0}$ there is a neighbourhood $V$ of zero in $A$ such that if $x \in V$ then $\left|p_{n}(x)\right| \leq \gamma_{n}$ for all $n \geq 0$. Finally, by the joint continuity of multiplication in $A$ there is a sequence $\left\{M_{n}\right\}_{n \geq l}$ of positive integers, and a sequence $\left\{\delta_{n}\right\}_{n \geq 1}$ of positive numbers such that if $\|x\|_{i}<\alpha$, $\|y\|_{i}<\beta$ for $1 \leq i \leq M_{n}$, where $\alpha \beta<\delta_{n}$, then $\|x y\|_{i}<1,1 \leq j \leq n$.

Let $\left\{\mu_{n}\right\}_{n \geq 1}$ be a sequence of positive numbers such that $\gamma_{n} \mu_{n}^{-1} \rightarrow 0$ as $n \rightarrow \infty$. Define inductively a sequence $\left\{x_{n}\right\}_{n \geq 1} \subseteq A$ such that

(i) $x_{n} \in U, D t \cdot x_{n} \in V$. 
(ii) $\left\|x_{n}\right\|_{i}<2^{-n} \delta_{n} \min _{\substack{1 \leq j \leq n \\ 1 \leq m \leq n}}\left\|t^{j}\right\|_{m}^{-1}$ for $1 \leq i \leq M_{n}$.

(iii) $\left|p_{k}\left(D x_{n}\right)\right| \geq \mu_{n+k}+k+\sum_{i=1}^{n+k+1} i \gamma_{n+k+1-i}+\sum_{i=1}^{n-1}\left|p_{i+k}\left(D x_{n-i}\right)\right|$.

It follows from (ii) that if $m>j$ then $\left\|t^{m-j} x_{m}\right\|_{i}<2^{-m}, 1 \leq i \leq m$, and so for each $j$ the series $\sum_{m>j} t^{m-j} x_{m}$ converges in $A$ to some element $y_{j}$. Set $y=y_{0}=\sum_{m \geq 1} t^{m} x_{m}$. Then

$$
\begin{aligned}
p_{n}(D y) & =p_{n} D\left\{t^{n+2} y_{n+2}+\sum_{i=1}^{n+2} t^{i} x_{i}\right\} \\
& =p_{n} D\left\{\sum_{i=1}^{n+2} t^{i} x_{i}\right\} \\
& =p_{n}\left\{D t \cdot x_{1}+\sum_{i=2}^{n+2} i t^{i-1} D t . x_{i}+\sum_{i=1}^{n+2} t^{i} D x_{i}\right\} \\
& =p_{n}\left(D t . x_{1}\right)+\sum_{j=0}^{n}\left\{\sum_{i=2}^{n+2} i p_{j}\left(t^{i-1}\right) p_{n-j}\left(D t . x_{i}\right)+\sum_{i=1}^{n+2} p_{j}\left(t^{i}\right) p_{n-j}\left(D x_{i}\right)\right\} \\
& =\sum_{i=1}^{n+1} i p_{n+1-i}\left(D t . x_{i}\right)+\sum_{i=0}^{n-1} p_{i}\left(D x_{n-i}\right) .
\end{aligned}
$$

Thus if $n \geq k+2$

$$
\left|p_{n}(D y)\right| \geq\left|p_{k}\left(D x_{n-k}\right)\right|-\sum_{i=1}^{n+1} i\left|p_{n+1-i}\left(D t . x_{i}\right)\right|-\left[\sum_{i=0}^{k-1}+\sum_{i=k+1}^{n-1}\right]\left|p_{i}\left(D x_{n-i}\right)\right| .
$$

But by (i) $\sum_{i=0}^{k-1}\left|p_{i}\left(D x_{n-i}\right)\right| \leq k$ and $\sum_{i=1}^{n+1} i\left|p_{n+1-i}\left(D t . x_{i}\right)\right| \leq \sum_{i=1}^{n+1} i \gamma_{n+1-i}$, and so by (iii) $\left|p_{n}(D y)\right| \geq \mu_{n}$.

Now choose a non-zero scalar $\lambda$ so small that $\lambda D y \in V$. Then for each $n \geq 0, \quad|\lambda| \mu_{n} \leq\left|p_{n}(\lambda D y)\right| \leq \gamma_{n}$, and so $\gamma_{n} \mu_{n}^{-1} \geq|\lambda|>0$. But this is impossible for all $n$ by the definition of the sequence $\left\{\mu_{n}\right\} ;$ and so the result follows. 
REMARK. The hypothesis of Theorem 1 is satisfied by any Banach algebra of power series in which the projections are continuous, for clearly the family $\left\{\left\|p_{n}\right\|^{-1} p_{n}\right\}_{n \geq 0}$ is equicontinuous. The algebra of entire functions on the complex plane, considered as a power series algebra, is another example. Cauchy's inequalities show easily that in this case the family $\left\{p_{n}\right\}_{n \geq 0}$ is equicontinuous.

In Newman [5] it is shown that there are no non-zero continuous derivations on a certain radical Banach algebra $R$, the elements of which are formal power series $\sum_{i \geq 1} a_{i} t^{i}$ with $\sum\left|a_{i}\right|\left|\lambda_{i}{ }^{i}\right|<\infty$ for a certain sequence $\left\{\lambda_{i}\right\}_{i \geq 1}$, this latter expression defining the norm. This algebra clearly satisfies the conditions of Theorem 1 , and we conclude that $R$ admits no non-zero derivations. This establishes the falsity of the conjecture of Singer and Wermer stated in [5], namely that a commutative Banach algebra which admits no non-zero derivations is necessary semisimple. Newman's result had shown it false if the derivations were restricted to being continuous. The conjecture is true for finite dimensional algebras by [2], Theorem 4.3.

The proof of Theorem 1 made use of the fact that for each $x \in A$ there is a positive number $K(x)$ such that $\left|\gamma_{n}^{-1} p_{n}(x)\right| \leq K(x)$ for all $n \geq 0$. If we use this condition as hypothesis we can obtain the following result, which requires no continuity restrictions on the projections.

THEOREM 2. Suppose that A satisfies the following two conditions:

(a) if $\sum a_{i} t^{i} \in A$ then $\sum_{i \geq n} a_{i} t^{i} \rightarrow 0$ in $A$ as $n \rightarrow \infty$;

(b) there is a sequence $\left\{\gamma_{n}\right\}$ of positive real numbers such that for each $x \in A$ there is $K(x)$ such that $\left|Y_{n}^{-1} p_{n}(x)\right| \leq K(x)$ for all $n \geq 0$.

Let $D$ be a derivation on $A$ with $D t=0$. Then $D=0$.

Proof. The proof follows the same line as that of Theorem, 1 and we give an outline only. 
Suppose $p_{k} D$ is discontinuous but that $p_{0} D, \ldots, p_{k-1} D$ are continuous. Let $U,\left\{\delta_{n}\right\},\left\{M_{n}\right\}$ be as in the proof of Theorem 1 , and let $\left\{\mu_{n}\right\}_{n \geq 1}$ be a sequence of positive numbers such that $\gamma_{n} \mu_{n}^{-1}+0$ as $n+\infty$. Define inductively a sequence $\left\{x_{n}\right\}_{n \geq 1} \subseteq A$ such that

$$
\begin{aligned}
& \text { (i)' } x_{n} \in U \text {. } \\
& \text { (ii)' }\left\|x_{n}\right\|_{i}<2^{-n} \delta_{n} \min _{\substack{1 \leq j \leq n \\
1 \leq m \leq n}}\left\|t^{j}\right\|_{m}^{-1} \text { for } 1 \leq i \leq M_{n} . \\
& \text { (iii)' }\left|p_{k}\left(D x_{n}\right)\right| \geq \mu_{n+k}+k+\sum_{i=1}^{n-1}\left|p_{i+k}\left(D x_{n-i}\right)\right| .
\end{aligned}
$$

Then $y=\sum_{m \geq 1} t^{m} x_{m} \in A$ and $\left|p_{n}(D y)\right| \geq \mu_{n}$. But then $\left|\gamma_{n}^{-1} p_{n}(D y)\right| \geq \gamma_{n}^{-1} \mu_{n} \rightarrow \infty$ as $n \rightarrow \infty$ by the definition of $\left\{\mu_{n}\right\}$ contradicting hypothesis $(b)$. It follows that $p_{n} D$ is continuous for all $n \geq 0$.

To see that $D=0$, suppose $D\left(\sum a_{i} t^{i}\right)=\sum b_{i} t^{i}$. Then for each $j$, $p_{j} D\left(\sum_{i \geq n} a_{i} t^{i}\right)=b_{j}$ for all $n$, and so by (a) it follows that $b_{j}=0$. Thus $D\left(\sum a_{i} t^{i}\right)=0$, as required.

\section{References}

[1] Richard Arens, "Linear topological division algebras", Buzz. Amer. Math. Soc. 53 (1947), 623-630.

[2] G. Hochschild, "Semisimple algebras and generalized derivations", Amer. J. Math. 64 (1942), 677-694.

[3] B.E. Johnson, "Continuity of derivations on commutative algebras", Amer. J. Math. 91 (1969), 1-10. 
[4] R.J. Loy, "A class of topological algebras of power series", Carleton Mathematical Series 11 (to appear).

[5] Donald J. Newman, "A radical algebra without derivations", Proc. Amer. Math. Soc. 10 (1959), 584-586.

Department of Mathematics,

Carleton University,

Ottawa, Canada. 